\title{
Inverse Optimal Control for Finite-Horizon Discrete-time Linear Quadratic Regulator Under Noisy Output
}

\author{
Han Zhang ${ }^{1}$, Xiaoming $\mathrm{Hu}^{1}$
}

\begin{abstract}
In this paper, the problem of inverse optimal control for finite-horizon discrete-time Linear Quadratic Regulators (LQRs) is considered. The goal of the inverse optimal control problem is to recover the corresponding objective function by the noisy observations. We consider the problem of inverse optimal control in two scenarios: 1) the distributions of the initial state and the observation noise are unknown, yet the exact observations on the initial states and the noisy observations on system output are available; 2) the exact observations on the initial states are not available, yet the observation noises are known white Gaussian and the distribution of the initial state is also Gaussian (with unknown mean and covariance). For the first scenario, we formulate the problem as a risk minimization problem and show that its solution is statistically consistent. For the second scenario, we fit the problem into the framework of maximum-likelihood and Expectation Maximization (EM) algorithm is used to solve this problem. The performance for the estimations are shown by numerical examples.
\end{abstract}

\section{INTRODUCTION}

The problem of inverse optimal control is first proposed by [11] and has found a multitude of applications in the field of robotics, economics and bionics [15], [6], [3]. It is well-known that the goal of a "forward" optimal control problem is to find the optimal control input as well as the optimal trajectory when the cost function, system dynamics and initial conditions are given. On the other hand, the inverse optimal control problem aims to find the parameters in the corresponding cost function, provided that the observations on the system outputs (which are contaminated by noises) are available and the system dynamics are known.

In this paper, the problem of inverse optimal control for discrete-time Linear Quadratic Regulators (LQRs) over finite-time horizon is considered. Namely, we aim to find the parameters in the quadratic objective funtion given the discrete-time linear system dynamics and observations of the noisy system output.

Inverse optimal control for LQR, particularly in the continuous infinite time-horizon case, has been studied by a number of authors [1], [8], [7]. They assume the optimal feedback gain $K$ is known exactly and focus on recovering the objective function. It was shown in [5] that the search for matrices $Q$ and $R$ can be formulated with Linear Matrix Inequalities (LMI) when the feedback gain $K$ is known. Nevertheless, in many scenarios, the feedback control gain $K$ can not be known exactly. In addition, we are interested in case of finite-time horizon, wherein the feedback gain $K_{t}$ is

\footnotetext{
*This work is supported by China Scholarship Council.

1 Both of the authors are with the Department of Mathematics, KTH Royal Institute of Technology, Sweden.
}

time-varying. [17] considers the discrete infinite time-horizon case with noisy observations, in which the optimal feedback gain $K$ is time-invariant. Their approach is to identify the feedback matrix $K$ first and solve for $Q$ and $R$ similar to the method proposed in [5]. Such an approach is not applicable in the finite-time horizon case, since the feedback gain $K_{t}$ is time-varying. Furthermore, the idea of "identify the feedback gain $K_{t}$, then compute the corresponding $Q$ " suffers from the huge number of parameters in the identification stage, i.e., the number of $K_{t}$ 's is proportional to the length of the timehorizon and the length of the time-horizon is usually large. In addition, such identification does not use the knowledge that the $K_{t}$ 's are actually generated by an LQR hence the performance of such method can not be guaranteed in the finite-time horizon case.

Spoken from a broader perspective, the problem of inverse optimal control has received considerable attention in the community. In particular, people have been focused on systems with nonlinear dynamics and objective functions composed by weighted-sum of base functions in recent years. In [12] and [4], the authors analyze the optimality conditions for the optimal control problem and a method based on minimizing the violation of these conditions is proposed. Nevertheless, as pointed out by [2], the approaches proposed in [12] and [4] are not statistically consistent and sensitive to observation noise. [2] present a statistically consistent formulation, but results in a difficult optimization problem. [14], [13] also consider the discrete finite time-horizon case. They consider the Pontryagin's Maximum Principle (PMP) for the optimal control problem and pose an optimization problem whose constraints are two of the three conditions of PMP; they then minimize the residual of the third PMP condition. In addition, they assume the optimal control input is known exactly while in our case, the estimation of the parameter relies on exact samples of initial values or noisy system output (only). The question of identifiability, i.e. uniqueness of the solution, for this approach is addressed, however, the statistical consistency of the estimation is not claimed. In a very recent work [10], the authors consider the discrete-time inverse optimal control problem for nonlinear systems when some segments of the trajectories and input observations are missing.

Without any doubt, the problem of inverse optimal control for $L Q R$ can be viewed as a special case of aforementioned problems for nonlinear systems. However, special structure of LQR is utilized and we are able to show the statistical consistency for the estimation. Furthermore, under Gaussian assumptions, we are able to use EM-algorithm to solve the 
problem due to its special structure. The performance of the algorithms are shown by numerical examples.

Notations: $\mathbb{S}_{+}^{n}$ denotes the cone of $n$ dimensional positive semi-definite matrices. $\mathbb{S}_{++}^{n}$ denotes the set of strictly positive semi-definite matrices. $\|\cdot\|_{F}$ denotes the Frobenius norm of a matrix. $\|\cdot\|$ denotes the $l_{2}$ norm of a vector. $\operatorname{tr}(\cdot)$ denotes the trace of a matrix and $\operatorname{tr}\left(G_{1}^{T} G_{1}\right)=\left\|G_{1}\right\|_{F}^{2}$. We denote $\otimes$ as the Kronecker product. The $i$ 'th row of matrix $G$ is denoted as $[G]_{i}$.

\section{Problem Formulation}

It is well-known that the "forward" optimal LQ problem reads

$$
\begin{aligned}
& \min _{x_{1: N}, u_{1: N-1}} J=x_{N}^{T} S x_{N}+\sum_{t=1}^{N-1}\left(u_{t}^{T} R u_{t}+x_{t}^{T} Q x_{t}\right) \\
& \text { s.t. } x_{t+1}=A x_{t}+B u_{t}, \quad x_{1}=\bar{x},
\end{aligned}
$$

where $S, Q \in \mathbb{S}_{+}^{n}, R \in \mathbb{S}_{++}^{m}, x_{t} \in \mathbb{R}^{n}$ and $u_{t} \in \mathbb{R}^{m}$.

Suppose we can observe the noisy output $y_{t}=C x_{t}+v_{t}$, where $v_{t} \in \mathbb{R}^{l}$ denotes the noise term, $y_{t} \in \mathbb{R}^{l}$ and the matrices $(A, B, C)$ are known. For simplicity, in this paper, we consider the case of $R=I$ and $S=0$. The inverse optimal control problem in this paper aims to find $Q$ in the objective function using:

1) the exact samples of initial value $x_{1}=\bar{x}$ and the noisy output $y_{2: N}$, or

2) the noisy output $y_{1: N}$ under Gaussian assumption.

In addition, it is assumed that $(A, B)$ is controllable and $B$ has full column rank. Moreover, we assume that $A$ is invertible. To see that the assumption is reasonable, consider a discrete-time system sampled from a continuous linear system $\dot{x}=\hat{A} x+\hat{B} u$, where the sample period $\Delta t$ is small. Hence for the discretized linear system, we have $A=e^{\hat{A} \Delta t}$, $B=\int_{0}^{\Delta t} e^{\hat{A} \tau} \hat{B} d \tau$. It is clear that $A=e^{\hat{A} \Delta t}$ is invertible.

\section{MAIN RESUlT}

\section{A. Inverse Optimal Control Using Exact Initial Values and Noisy Output}

Inspired by [2], we first formulate the inverse optimal control problem when exact samples of the initial Value $x_{1}=$ $\bar{x}$ and noisy output $y_{2: N}$ are available. Suppose the probability space $(\Omega, \mathcal{F}, \mathbb{P})$ carries independent random vectors $\bar{x} \in$ $\mathbb{R}^{n},\left\{v_{t} \in \mathbb{R}^{n}\right\}_{t=2}^{N}$ distributed according to some unknown distributions. The following assumptions are made in the remainder of this section:

Assumption 1. $\mathbb{E}\left(\|\bar{x}\|^{2}\right)<+\infty, \mathbb{E}\left(v_{t}\right)=0$ and $\mathbb{E}\left(\left\|v_{t}\right\|^{2}\right)<$ $+\infty$.

Assumption 2. $\forall \eta \in \mathbb{R}^{n}, \exists r(\eta) \in \mathbb{R} \backslash\{0\}$ such that $\mathbb{P}\left(\bar{x} \in \mathscr{B}_{\varepsilon}(r \eta)\right)>0, \forall \varepsilon>0$, where $\mathscr{B}_{\varepsilon}(r \eta)$ is the open $\varepsilon$-ball centered at $r \eta$.

Assumption 3. The discrete-time linear system defined by $(A, B, C)$ is a square-system, i.e., $l=m$ and has relative degree [19] $\left(r_{1}, \cdots, r_{m}\right)$.
Assumption 4. The "real" $\bar{Q}$ belongs to a compact set $\overline{\mathbb{S}}_{+}^{n}(\varphi)=\left\{Q \in \mathbb{S}_{+}^{n} \mid\|Q\|_{F}^{2} \leq \varphi\right\}$.

With the assumptions above, we see that when solving the "forward" optimal LQ problem, the initial value $x_{1}$ used is actually a realization of the random vector $\bar{x}$, i.e., $x_{1}=\bar{x}(\omega)$; hence the "forward" LQR problem can actually be seen as

$$
\begin{gathered}
\min _{u_{1: N-1}(\omega), x_{2: N}(\omega)}\left\{J\left(u_{1: N-1}(\omega), x_{2: N}(\omega) ; Q ; \bar{x}(\omega)\right)\right. \\
\mid 2, \text { given } \omega \in \Omega\},
\end{gathered}
$$

Note that the optimal control input and trajectory $\left\{u_{t}^{*}\right\},\left\{x_{t}^{*}\right\}$ are now random vectors implicitly determined by the random variable $\bar{x}$ and the parameter $Q$. With the formulation of the "forward problem" (3), we can define the risk function

$$
\mathscr{R}(Q)=\mathbb{E}_{\xi}[f(Q ; \xi)]
$$

where $\xi=\left[\bar{x}^{T}, Y^{T}\right]^{T}, Y=\left[y_{2}^{T}, \cdots, y_{N}^{T}\right]^{T}$ and $f: \overline{\mathbb{S}}_{+}^{n}(\varphi) \times$ $\mathbb{R}^{n+(N-1) l} \mapsto \mathbb{R}$,

$$
f(Q ; \xi)=\sum_{t=2}^{N}\left\|y_{t}-C x_{t}^{*}(Q ; \bar{x})\right\|^{2}
$$

and $x_{2: N}^{*}(Q ; \bar{x})$ is the optimal trajectory to 3 .

In order to solve the inverse optimal control problem, it is rational to minimize the risk function, namely,

$$
\min _{Q \in \overline{\mathbb{S}}_{+}^{n}(\varphi)} \mathscr{R}(Q)
$$

Nevertheless, since the distributions of $\bar{x}$ and $v_{2: N}$ are unknown, it is not possible to solve (6) directly. However, (4) in principle, can be approximated by

$$
\mathscr{R}_{M}(Q)=\frac{1}{M} \sum_{i=1}^{M} f\left(Q ; \xi^{(i)}\right)
$$

where $\xi^{(i)}$ are i.i.d random samples. The statistical consistency for this approximation will be shown later.

On the other hand, by Pontryagin's Maximum Principle (PMP), if $u_{1: N-1}^{*}$ and $x_{1: N}^{*}$ are the optimal control and corresponding trajectory, then there exists adjoint variables $\lambda_{2: N}^{*}$ such that

$$
\begin{aligned}
& x_{t+1}^{*}=A x_{t}^{*}+B u_{t}^{*}, t=1: N-1 \\
& \lambda_{t}^{*}=A^{T} \lambda_{t+1}^{*}+Q x_{t}^{*}, t=2: N-1, \\
& \lambda_{N}^{*}=0 \\
& u_{t}=-B^{T} \lambda_{t+1}^{*}, t=1: N-1 .
\end{aligned}
$$

Although PMP in general provides necessary conditions for optimality, note that it becomes also sufficient conditions for optimality since the solution to the "forward" LQR problem is unique. Hence we can express $x_{2: N}^{*}$ in (5) using (8). Thus 
the approximated risk minimization problem reads

$$
\begin{aligned}
\min _{Q \in \overline{\mathbb{S}}_{+}^{n}(\varphi), x_{2: N}^{(i)}, \lambda_{2: N}^{(i)}} & \mathscr{R}_{M}^{x}(Q)=\frac{1}{M} \sum_{i=1}^{M} f\left(Q ; \xi^{(i)}\right) \\
\text { s.t. } \quad & x_{t+1}^{(i)}=A x_{t}^{(i)}-B B^{T} \lambda_{t+1}^{(i)}, \quad t=2: N-1, \\
\lambda_{t}^{(i)} & =A^{T} \lambda_{t+1}^{(i)}+Q x_{t}^{(i)}, t=2: N-1, \\
x_{2}^{(i)} & =A \bar{x}^{(i)}-B B^{T} \lambda_{2}^{(i)}, \\
\lambda_{N}^{(i)} & =0, \quad i=1: M .
\end{aligned}
$$

The superscript "star" is omitted in the above notation to avoid the confusion with the optimizer of (9). Note that the optimizer $\left(Q_{M}^{*}(\omega), x_{2: N}^{(i) *}(\omega), \lambda_{2: N}^{(i) *}(\omega)\right)$ is stochastic and is defined in the sense that it optimizes (7) for every $\omega \in \Omega$.

On the other hand, recall that $x_{t+1}^{*}=\left(A+B K_{t}(Q)\right) x_{t}^{*}$, where $K_{t}(Q)=-\left(B^{T} P_{t+1}(Q) B+I\right)^{-1} B^{T} P_{t+1}(Q) A$ and $P_{2: N}(Q)$ is the solution to Discrete-time Riccati Equation (DRE). Before continuing, we would like to present the following lemma:

Lemma III.1. ([21]) If $(A, B)$ is controllable, $A$ is invertible, $B$ has full column rank and $N \geq n+2, Q_{1} \neq Q_{2}, Q_{1}, Q_{2} \in$ $\mathbb{S}_{+}^{n}$, then the control gain $K_{t}\left(Q_{1}\right) \neq K_{t}\left(Q_{2}\right), t=1: N-1$.

Theorem III.1. Suppose $Q_{M}^{*} \in \overline{\mathbb{S}}_{+}^{n}(\varphi),\left\{x_{2: N}^{(i) *}\right\}$ and $\left\{\lambda_{2: N}^{(i) *}\right\}$ solves (9) and $N \geq n+2$, then $Q_{M}^{*} \stackrel{p}{\rightarrow} \bar{Q}$, where $\bar{Q}$ is the true value used in the "forward" problem (3).

Proof. Denote $z_{t}=\left(x_{t}^{T}, \lambda_{t}^{T}\right)^{T}, t=2: N$, then the first two constraints in (8) can be written as the following implicit dynamics

$$
\underbrace{\left[\begin{array}{cc}
I & B B^{T} \\
0 & A^{T}
\end{array}\right]}_{E} z_{t+1}=\underbrace{\left[\begin{array}{cc}
A & 0 \\
-Q & I
\end{array}\right]}_{F} z_{t}, \quad t=2: N-1 .
$$

Therefore, we can rewrite $(8)$ in the following compact matrix form as

$$
\underbrace{\left[\begin{array}{cccc}
\tilde{E} & & & \tilde{F} \\
-F & E & & \\
& \ddots & \ddots & \\
& & -F & E
\end{array}\right]}_{\mathscr{F}(Q)} \underbrace{\left[\begin{array}{c}
z_{2} \\
\vdots \\
z_{N}
\end{array}\right]}_{Z}=\underbrace{\left[\begin{array}{c}
A \bar{x}^{(i)} \\
0 \\
\vdots \\
0
\end{array}\right]}_{b(\bar{x})},
$$

where

$$
\tilde{E}=\left[\begin{array}{cc}
I & B B^{T} \\
0 & 0
\end{array}\right], \tilde{F}=\left[\begin{array}{ll}
0 & 0 \\
0 & I
\end{array}\right] .
$$

We claim that $\mathscr{F}(Q)$ is invertible. The easiest way to see this is that for an arbitrary $Q \in \mathbb{S}_{+}^{n},[10$ ) is a sufficient and necessary condition for the corresponding "forward" $L Q R$ problem. Since the "forward" LQR problem has a unique solution, it must hold that $\mathscr{F}(Q)$ is invertible for all $Q \in$ $\mathbb{S}_{+}^{n}$. Thus, it follows that $Z=\mathscr{F}(Q)^{-1} b(\bar{x})=\mathscr{F}(Q)^{-1} \tilde{A} \bar{x}$, where $\tilde{A}=\left[A^{T}, 0, \cdots, 0\right]^{T}$. Hence $f(Q ; \xi)$ can be rewritten as

$$
f(Q ; \xi)=\|Y-G Z\|^{2}=\left\|Y-G \mathscr{F}(Q)^{-1} \tilde{A} \bar{x}\right\|^{2}
$$

where $G=I_{N-1} \otimes\left[C, 0_{l \times n}\right]$ and $Y=\left[y_{2}^{T}, \cdots, y_{N}^{T}\right]^{T}$.

It is clear that $f(Q ; \xi)$ is continuous with respect to $\xi$, hence it is a measurable function of $\xi$ at each $Q$. Further, $\mathscr{F}(Q)$ is continuous and hence $\mathscr{F}(Q)^{-1}$ is continuous. Then $f(Q ; \xi)$ is also continuous with respect to $Q$.

On the other hand, since $\mathscr{F}(Q)^{-1}$ is continuous and $Q$ lives in a compact set, then $\left\|\mathscr{F}(Q)^{-1}\right\|_{F}$ is bounded, i.e., $\left\|\mathscr{F}(Q)^{-1}\right\|_{F} \leq \bar{\varphi}$ for some finite positive $\bar{\varphi}$. It follows that $\mathbb{E}\left(\left\|Z^{*}\right\|^{2}\right)=\mathbb{E}\left(\left\|\mathscr{F}(\bar{Q})^{-1} \tilde{A} \bar{x}\right\|^{2}\right) \leq$ $\left\|\mathscr{F}(\bar{Q})^{-1}\right\|_{F}^{2}\|\tilde{A}\|_{F}^{2} \mathbb{E}\left(\|\bar{x}\|^{2}\right)<+\infty$, where $Z^{*}$ corresponds to the "true" $\bar{Q}$.

Recall that $y_{t}=C x_{t}^{*}+v_{t}$ and this implies that $Y=G Z^{*}+\zeta$, where $\zeta=\left[v_{2}^{T} \cdots, v_{N}^{T}\right]^{T}$. By Assumption 1, $\mathbb{E}\left(\left\|v_{t}\right\|^{2}\right)<\infty$, which implies $\mathbb{E}\left(\|\zeta\|^{2}\right)<+\infty$. Therefore, $\mathbb{E}\left(\|Y\|^{2}\right)=\mathbb{E}\left(\| G Z^{*}+\right.$ $\left.\zeta \|^{2}\right) \leq 2\left(\mathbb{E}\left(\left\|G Z^{*}\right\|^{2}\right)+\mathbb{E}\left(\|\zeta\|^{2}\right)\right) \leq 2\left(\|G\|_{F}^{2} \mathbb{E}\left(\left\|Z^{*}\right\|^{2}\right)\right.$ $\left.+\mathbb{E}\left(\|\zeta\|^{2}\right)\right)<+\infty$. Hence it holds that

$$
\begin{aligned}
f(Q, \xi) & =\left\|Y-G \mathscr{F}(Q)^{-1} \tilde{A} \bar{x}\right\|^{2} \\
& \leq 2\left(\|Y\|^{2}+\left\|G \mathscr{F}(Q)^{-1} \tilde{A} \bar{x}\right\|^{2}\right) \\
& \leq 2\left(\|Y\|^{2}+\|G\|_{F}^{2}\left\|\mathscr{F}(Q)^{-1}\right\|_{F}^{2}\|\tilde{A}\|_{F}^{2}\|\bar{x}\|^{2}\right) \\
& \leq 2\left(\|Y\|^{2}+\bar{\varphi}^{2}\|G\|_{F}^{2}\|\tilde{A}\|_{F}^{2}\|\bar{x}\|^{2}\right):=d(\xi),
\end{aligned}
$$

and it is clear that $\mathbb{E}(d(\xi))<+\infty$ since $\mathbb{E}\left(\|Y\|^{2}\right)<+\infty$ and $\mathbb{E}\left(\|\bar{x}\|^{2}\right)<+\infty$. By the analysis above, we conclude that the uniform law of large numbers [9] applies, namely,

$$
\sup _{Q \in \overline{\mathbb{S}}_{+}^{n}(\varphi)}\left\|\frac{1}{M} \sum_{i=1}^{M} f\left(Q, \xi^{(i)}\right)-\mathbb{E}_{\xi}[f(Q ; \xi)]\right\| \stackrel{p}{\rightarrow} 0 .
$$

Besides (11), if we are able to show $\bar{Q}$ is the unique optimizer to (6), then $Q_{M}^{*} \stackrel{p}{\rightarrow} \bar{Q}$ follows directly from Theorem 5.7 in [20].

Note that by assumption, $\bar{x},\left\{v_{t}\right\}$ are independent, hence $x_{t}^{*}(Q ; \bar{x})$ are independent of the noises $\left\{v_{t}\right\}$. Since $y_{t}=$ $C x_{t}^{*}(\bar{Q}, \bar{x})+v_{t}, \mathbb{E}\left(v_{t}\right)=0, t=2: N$, (4) can be simplified as $\mathscr{R}(Q)=L(Q)+\sum_{t=2}^{N} \mathbb{E}\left(\left\|v_{t}\right\|^{2}\right)$, where $L(Q)=$ $\mathbb{E}\left(\sum_{t=2}^{N}\left\|\bar{y}_{t}(\bar{Q}, \bar{x})-\bar{y}_{t}(Q, \bar{x})\right\|^{2}\right), \bar{y}_{t}(\bar{Q}, \bar{x})=C x_{t}^{*}(\bar{Q}, \bar{x})$ and $\bar{y}_{t}(Q, \bar{x})=C x_{t}^{*}(Q, \bar{x})$. It is clear that $Q=\bar{Q}$ minimizes the risk function $\mathscr{R}(Q)$. What remains to show is the uniqueness.

By Assumption 3, the system has relative degree $\left(r_{1}, \cdots, r_{m}\right)$ and it holds by definition that

$$
\begin{aligned}
& c_{i} A^{j} B=0, j=0: r_{i}-2, \quad c_{i} A^{r_{i}-1} B \neq 0, \\
& \mathscr{L}=\left[\begin{array}{c}
c_{1} A^{r_{1}-1} B \\
\vdots \\
c_{m} A^{r_{m}-1} B
\end{array}\right] \text { is nonsingular, }
\end{aligned}
$$

where $c_{i}$ denotes the $i$ 'th row of $C$. Therefore it follows that

$$
\begin{aligned}
\bar{y}_{t+1, i}(Q, \bar{x}) & =c_{i} \Pi_{j=1}^{t}\left(A+B K_{j}\right) \bar{x} \\
& =c_{i} A^{t} \bar{x}, \quad t=1: r_{i}-1, \quad i=1: m . \\
\bar{y}_{r_{i}+1, i}(Q, \bar{x}) & =c_{i} A^{r_{i}} \bar{x}+c_{i} A^{r_{i}-1} B K_{1}(Q) \bar{x} .
\end{aligned}
$$


Note that

$$
\begin{aligned}
& \mathscr{R}(Q) \geq L(Q)=\sum_{t=2}^{N} \mathbb{E}\left[\left\|\bar{y}_{t}(\bar{Q}, \bar{x})-\bar{y}_{t}(Q, \bar{x})\right\|^{2}\right] \\
& =\sum_{i=1}^{m} \sum_{t=2}^{N} \mathbb{E}\left[\left\|\bar{y}_{t, i}(\bar{Q}, \bar{x})-\bar{y}_{t, i}(Q, \bar{x})\right\|^{2}\right] \\
& \geq \sum_{i=1}^{m} \mathbb{E}\left[\left\|\bar{y}_{r_{i}+1, i}(\bar{Q}, \bar{x})-\bar{y}_{r_{i}+1, i}(Q, \bar{x})\right\|^{2}\right] \\
& =\mathbb{E}\left[\left\|\mathscr{L}\left(K_{1}(\bar{Q})-K_{1}(Q)\right) \bar{x}\right\|^{2}\right]
\end{aligned}
$$

By Lemma III.1. we know that if $Q \neq \bar{Q}$, then $K_{1}(Q) \neq$ $K_{1}(\bar{Q})$. Hence there exists $\eta \in \mathbb{R}^{n}$, such that $K_{1}(\bar{Q}) \eta \neq$ $K_{1}(Q) \eta$. On the other hand, by Assumption $2, \exists r(\eta) \neq 0$, such that $\mathbb{P}\left(\bar{x} \in \mathscr{B}_{\varepsilon}(r \eta)\right)>0, \forall \varepsilon$. Since $r \neq 0$ and $\mathscr{L}$ is nonsingular, it follows that $\mathscr{L} K_{1}(Q)(r \eta) \neq \mathscr{L} K_{1}(\bar{Q})(r \eta)$. Furthermore, since $\mathscr{L} K_{1}(Q) \eta$ is continuous with respect to $\eta, \forall Q \in \mathbb{S}_{+}^{n}$, this implies $\exists \varepsilon_{1}$, such that $\mathscr{L} K_{1}(Q) \bar{x} \neq$ $\mathscr{L} K_{1}(\bar{Q}) \bar{x}, \forall \bar{x} \in \mathscr{B}_{\varepsilon_{1}}(r \eta)$ and $\mathbb{P}\left(\bar{x} \in \mathbb{B}_{\varepsilon_{1}}(r \eta)\right)>0$. Thus $\mathscr{R}(Q) \geq \int_{\mathscr{B}_{\varepsilon_{1}}(r \eta)}\left\|\mathscr{L}\left(K_{1}(Q)-K_{1}(\bar{Q})\right) \bar{x}(\omega)\right\|^{2} \mathbb{P}(d \omega)>$ 0 . Hence $\bar{Q}$ is the unique minimizer to (4) and the statement follows.

We have shown that the risk minimization problem (9) is statistically consistent. As stated in the proof, the (9) can be rewritten in the compact form of $\min _{Q \in \overline{\mathbb{S}}_{+}^{n}(\varphi)} \frac{1}{M} \sum_{i=1}^{M}\left\|Y^{(i)}-G \mathscr{F}(Q)^{-1} \tilde{A} \bar{x}\right\|^{2}$. To solve the problem, we introduce the following convex matrix function $\hat{f}_{\varepsilon}: \mathbb{S}^{n} \mapsto \mathbb{R}, \hat{f}_{\varepsilon}(Q)=\varepsilon \ln \operatorname{tr}\left(e^{Q / \varepsilon}\right)=\varepsilon \ln \left[\sum_{i=1}^{N} e^{\sigma_{i}(Q) / \varepsilon}\right]$ [16], where $\sigma_{i}(Q)$ is the $i$ 'th largest eigenvalue of $Q$. It holds that $\sigma_{1}(Q) \leq \hat{f}_{\varepsilon}(Q) \leq \sigma_{1}(Q)+\varepsilon \ln n$. Hence when $\varepsilon$ is small, the function $\hat{f}(Q)$ approximates the largest eigenvalue of $Q$ well. On the other hand, the gradient of $\hat{f}_{\varepsilon}(Q)$ reads $\nabla_{Q} \hat{f}_{\varepsilon}(Q)=\left[\sum_{i=1}^{N} e^{\sigma_{i}(Q) / \varepsilon}\right]^{-1}\left[\sum_{i=1}^{N} e^{\sigma_{i}(Q) / \varepsilon} \nu_{i} \nu_{i}^{T}\right]$, where $\left(\sigma_{i}(Q), \nu_{i}\right)$ are eigen-pairs of $Q$ with $\left\|\nu_{i}\right\|=1, \forall i$. Note that for $\varepsilon$ small enough, the gradient only numerically depends on the eigenvectors that correspond to the largest eigenvalues [16], which makes the gradient easy to compute. With the setup above, we approximate the semi-positive definite constraint $Q \in \mathbb{S}_{+}^{n}$ with $\hat{f}_{\varepsilon}(-Q) \leq 0$ and we can solve the optimization problems with standard nonlinear optimization solvers.

\section{B. Inverse Optimal Control Using Noisy Output Under Gaussian Assumption}

In Section III-A it is assumed that the distributions of the noise and the initial value are unknown, yet we can get exact samples of the initial value. But in some scenarios, the exact sample on initial value is not available and we can only observe the noisy measurement $y_{1}=C x_{1}+v_{1}$. In this scenario, we have the following assumption.

Assumption 5. $\bar{x}=x_{1} \sim \mathcal{N}\left(m_{1}, \Sigma_{1}\right), v_{t} \sim \mathcal{N}\left(0, \Sigma_{v}\right)$ and $v_{1: N}$ are white. $\Sigma_{v}$ is known, but $m_{1}$ and $\Sigma_{1}$ is not apriori known.

Recall the proof of Theorem III.1, we can write $Y=$ $\left[y_{2}^{T}, \cdots, y_{N}^{T}\right]^{T}=G \mathscr{F}(Q)^{-1} \tilde{A} \bar{x}+\left[v_{2}^{T}, \cdots, v_{N}^{T}\right]^{T}$. More precisely, it holds that

$$
y_{t}=G_{t} \mathscr{F}(Q)^{-1} \tilde{A} \bar{x}+v_{t}, t=2: N,
$$

where $G_{t}=[G]_{l(t-1)+1: l t}$. We can regard $y_{1: N}$ as the output of the following system:

$$
\begin{aligned}
& \eta_{t+1}=\eta_{t}, t=1: N-1, \eta_{1}=\bar{x} \\
& y_{t}=\underbrace{G_{t} \mathscr{F}(Q)^{-1} \tilde{A}}_{\tilde{C}_{t}(Q)} \eta_{t}+v_{t}, t=1: N,
\end{aligned}
$$

where $\tilde{C}_{1}(Q)=C$ and $\tilde{C}_{t}(Q)=G_{t} \mathscr{F}(Q)^{-1} \tilde{A}, t=2$ : $N$. Now, under Assumption 5 , the inverse optimal control problem can be viewed as a system identification problem for Linear Gaussian Model, which can be solved using maximum log-likelihood method. If there are $M$ output sequences available, then it means we have $M$ i.i.d samples $x_{1}^{(1: M)}$ of the initial value. Since $v_{t}^{(1: M)}$ is also i.i.d., $y_{t}^{(i)}$ and $y_{t}^{(j)}$ are independent for all $i \neq j$. We would like to use EMalgorithm to solve the maximum log-likelihood problem.

Note that in (12), all of the stochasticity comes from the inital value $\eta_{1}=\bar{x}$ as well as the output measurements $y_{1: N}$, hence we choose $\eta_{1}$ to be the latent variable and let $\theta$ parametrize $Q, m_{1}$ and $\Sigma_{1}$. We now compute the auxiliary function $\mathcal{Q}\left(\theta, \theta_{k}\right)$ of the EM-algorithm.

Proposition III.1. Under Assumption 5 the auxiliary function $\mathcal{Q}\left(Q, Q_{k}\right)$ that corresponds to the model (12) is given by

$$
\mathcal{Q}\left(\theta, \theta_{k}\right) \propto \mathcal{Q}_{1}\left(\theta, \theta_{k}\right)+\mathcal{Q}_{2}\left(\theta, \theta_{k}\right)
$$

where

$$
\begin{aligned}
& \mathcal{Q}_{1}\left(\theta, \theta_{k}\right)=-\sum_{i=1}^{M}\left\{\ln \operatorname{det} \Sigma_{1}+\operatorname{tr}\left[\Sigma_{1}^{-1} P_{1 \mid N}^{(i)}\right]\right. \\
& \left.+\sum_{t=2}^{N} \operatorname{tr}\left[\Sigma_{1}^{-1}\left(\hat{\eta}_{1 \mid N}^{(i)}-m_{1}\right)\left(\hat{\eta}_{1 \mid N}^{(i)}-m_{1}\right)^{T}\right]\right\} \\
& \mathcal{Q}_{2}\left(\theta, \theta_{k}\right)=-\sum_{i=1}^{M}\left\{\sum_{t=2}^{N} \operatorname{tr}\left[\Sigma_{v}^{-1} \tilde{C}_{t}(Q) P_{1 \mid N}^{(i)} \tilde{C}_{t}(Q)^{T}\right]\right. \\
& \left.+\sum_{t=2}^{N} \operatorname{tr}\left[\Sigma_{v}^{-1}\left(y_{t}^{(i)}-\tilde{C}_{t}(Q) \hat{\eta}_{1 \mid N}^{(i)}\right)\left(y_{t}^{(i)}-\tilde{C}_{t}(Q) \hat{\eta}_{1 \mid N}^{(i)}\right)^{T}\right]\right\}
\end{aligned}
$$

and $\hat{\eta}_{1 \mid N}^{(i)}:=\mathbb{E}_{\theta_{k}}\left[\eta_{1}^{(i)} \mid y_{1: N}^{(i)}\right], P_{1 \mid N}^{(i)}=\operatorname{cov}_{\theta_{k}}\left[\eta_{1}^{(i)} \mid y_{1: N}^{(i)}\right]$.

Proof. By Bayes' rule, it follows that

$$
p_{\theta}\left(\eta_{1}^{(1: M)}, y_{1: N}^{(1: M)}\right)=\prod_{i=1}^{M} p_{\theta}\left(\eta_{1}^{(i)}\right) \prod_{t=1}^{N} p_{\theta}\left(y_{t}^{(i)} \mid \eta_{1}\right)
$$

Hence by the definition of the auxiliary function $\mathcal{Q}\left(Q, Q_{k}\right)$, 
we have

$$
\begin{aligned}
& \mathcal{Q}\left(\theta, \theta_{k}\right) \\
& =\int \ln p_{\theta}\left(\eta_{1}^{(1: M)}, y_{1: N}^{(1: M)}\right) p_{\theta_{k}}\left(\eta_{1}^{(1: M)} \mid y_{1: N}^{(1: M)}\right) d \eta_{1}^{(1: M)} \\
& =\int\left(\sum_{i=1}^{M} \ln p_{\theta}\left(\eta_{1}^{(i)}, y_{1: N}^{(i)}\right)\right) \prod_{i=1}^{M} p_{\theta_{k}}\left(\eta_{1}^{(i)} \mid y_{1: N}^{(1: M)}\right) d \eta_{1}^{(1: M)} \\
& =\sum_{i=1}^{M} \int \ln p_{\theta}\left(\eta_{1}^{(i)}, y_{1: N}^{(i)}\right) p_{\theta_{k}}\left(\eta_{1}^{(i)} \mid y_{1: N}^{(i)}\right) d \eta_{1}^{(i)} \\
& =\sum_{i=1}^{M}\left\{\int \ln p_{\theta}\left(\eta_{1}^{(i)}\right) p_{\theta_{k}}\left(\eta_{1}^{(i)} \mid y_{1: N}^{(i)}\right) d \eta_{1}^{(i)}\right. \\
& \left.+\sum_{t=1}^{N} \int \ln p_{\theta}\left(y_{t}^{(i)} \mid \eta_{1}^{(i)}\right) p_{\theta_{k}}\left(\eta_{1}^{(i)} \mid y_{1: N}^{(i)}\right) d \eta_{1}^{(i)}\right\}
\end{aligned}
$$

Note that $y_{1}^{(i)}=C \eta_{1}^{(i)}+v_{1}^{(i)}$, hence $p_{\theta}\left(y_{1}^{(i)} \mid \eta_{1}^{(i)}\right) \sim$ $\mathcal{N}\left(C \eta_{1}^{(i)}, \Sigma_{v}\right)$ and it is irrelevant with respect to $\theta$. By using the property of matrix trace and the fact of $\mathbb{E}_{\theta_{k}}\left[\eta_{1}^{(i)} \eta_{1}^{(i) T} \mid y_{1: N}^{(i)}\right]=\hat{\eta}_{1 \mid N}^{(i)} \hat{\eta}_{1 \mid N}^{(i) T}+P_{1 \mid N}^{(i)}$, also by ignoring the constant and irrelevant terms, it follows that

$$
\begin{aligned}
& \mathcal{Q}\left(\theta, \theta_{k}\right) \\
& \propto-\sum_{i=1}^{M}\left\{\ln \operatorname{det} \Sigma_{1}+\int\left\|\eta_{1}^{(i)}-m_{1}\right\|_{\Sigma_{1}^{-1}}^{2} p_{\theta_{k}}\left(\eta_{1}^{(i)} \mid y_{1: N}^{(i)}\right) d \eta_{1}^{(i)}\right. \\
& \left.+\sum_{t=2}^{N} \int\left\|y_{t}^{(i)}-\tilde{C}_{t}(Q) \eta_{1}^{(i)}\right\|_{\Sigma_{v}^{-1}}^{2} p_{\theta_{k}}\left(\eta_{1}^{(i)} \mid y_{1: N}^{(i)}\right) d \eta_{1}^{(i)}\right\} \\
& =\mathcal{Q}_{1}\left(\theta, \theta_{k}\right)+\mathcal{Q}_{2}\left(\theta, \theta_{k}\right) .
\end{aligned}
$$

Note that $\hat{\eta}_{1}^{(i)}$ and $P_{1 \mid N}^{(i)}$ can be obtained by fixed point smoothing [18]. We now check (13) and (14) in detail. In each iteration of EM-algorithm, we need to maximize $\mathcal{Q}\left(\theta, \theta_{k}\right)$ with respect to $\theta$. Note that $m_{1}$ and $\Sigma_{1}$ only show up in $\mathcal{Q}_{1}\left(\theta, \theta_{k}\right) ; Q$ shows up only in $\mathcal{Q}_{2}\left(\theta, \theta_{k}\right)$, therefore we can optimize them separately. Consider the derivative of $\mathcal{Q}_{1}\left(\theta, \theta_{k}\right)$ with respect to $m_{1}$ and $\Sigma_{1}$, the first order optimality condition for maximizing $\mathcal{Q}_{1}\left(\theta, \theta_{k}\right)$ reads:

$$
\begin{aligned}
& \partial_{m_{1}} \mathcal{Q}_{1}\left(\theta^{*}, \theta_{k}\right) \\
& =-\partial_{m_{1}}\left(\sum_{i=1}^{M}\left(m_{1}^{*}-\hat{\eta}_{1 \mid N}^{(i)}\right)^{T} \Sigma_{1}^{*-1}\left(m_{1}^{*}-\hat{\eta}_{1 \mid N}^{(i)}\right)\right) \\
& =-2 \sum_{i=1}^{M} \Sigma_{1}^{*-1}\left(m_{1}^{*}-\hat{\eta}_{1 \mid N}^{(i)}\right)=0, \\
& \partial_{\Sigma_{1}} \mathcal{Q}_{1}\left(\theta^{*}, \theta_{k}\right)=-\sum_{i=1}^{M}\left\{\Sigma_{1}^{*-1}-\Sigma_{1}^{*-1} P_{1 \mid N}^{(i)} \Sigma_{1}^{*-1}\right. \\
& \left.-\Sigma_{1}^{*-1}\left(m_{1}^{*}-\hat{\eta}_{1 \mid N}^{(i)}\right)\left(m_{1}^{*}-\hat{\eta}_{1 \mid N}^{(i)}\right)^{T} \Sigma_{1}^{*-1}\right\}=0 .
\end{aligned}
$$

Solving (16) and (17), we get

$$
m_{1}^{*}=\frac{1}{M} \sum_{i=1}^{M} \eta_{1 \mid N}^{(i)}
$$

$$
\begin{aligned}
& \Sigma_{1}^{-1} \sum_{i=1}^{M}\left[\Sigma_{1}-\left(m_{1}^{*}-\hat{\eta}_{1 \mid N}^{(i)}\right)\left(m_{1}^{*}-\hat{\eta}_{1 \mid N}^{(i)}\right)^{T}-P_{1 \mid N}^{(i)}\right] \Sigma_{1}^{-1}=0, \\
& \Leftrightarrow \sum_{i=1}^{M}\left[\Sigma_{1}-\left(m_{1}^{*}-\hat{\eta}_{1 \mid N}^{(i)}\right)\left(m_{1}^{*}-\hat{\eta}_{1 \mid N}^{(i)}\right)^{T}-P_{1 \mid N}^{(i)}\right]=0 \\
& \Leftrightarrow \Sigma_{1}^{*}=\frac{1}{M} \sum_{i=1}^{M}\left[\left(m_{1}^{*}-\hat{\eta}_{1 \mid N}^{(i)}\right)\left(m_{1}^{*}-\hat{\eta}_{1 \mid N}^{(i)}\right)^{T}+P_{1 \mid N}^{(i)}\right] .
\end{aligned}
$$

Note that 18 and 19 is the unique solution to the first order necessary optimality condition of maximizing $\mathcal{Q}_{1}\left(\theta, \theta_{k}\right)$. Hence it is the unique global maximizer for $\mathcal{Q}_{1}\left(\theta, \theta_{k}\right)$. On the other hand, we can not write the analytic solution for the optimizer $Q^{*}$ of maximizing $\mathcal{Q}_{2}\left(\theta, \theta_{k}\right)$ and if written in a compact from, the problem of maximizing $\mathcal{Q}_{2}\left(\theta, \theta_{k}\right)$ reads

$$
\begin{aligned}
\min _{Q \in \overline{\mathbb{S}}_{+}^{n}(\varphi)} & \sum_{i=1}^{M}\left\{\left\|Y^{(i)}-G \mathscr{F}(Q)^{-1} \tilde{A} \hat{\eta}_{1 \mid N}^{(i)}\right\|_{\Sigma_{V}^{-1}}^{2}\right. \\
& \left.+\sum_{t=1}^{N} \operatorname{tr}\left[\Sigma_{v}^{-1} G_{t} \mathscr{F}(Q)^{-1} \tilde{A} P_{1 \mid N}^{(i)} \tilde{A}^{T} \mathscr{F}(Q)^{-T} G_{t}^{T}\right]\right\},
\end{aligned}
$$

where $Y^{(i)}=\left[y_{2}^{(i) T}, \cdots, y_{N}^{(i) T}\right]^{T}, \Sigma_{V}=I_{N-1} \otimes \Sigma_{v}$. Using the function $\hat{f}_{\varepsilon}$ proposed by [16] and apply the same "trick" mentioned in the end of Section III-B, we can use standard nonlinear optimization solvers to solve (20).

\section{NUMERICAL EXAMPLES}

We first illustrate the performance of the estimation statistically when exact samples of initial value $x_{1}=\bar{x}$ are available. We consider a group of discrete-time linear systems sampled from continuous linear systems $\dot{x}=\hat{A} x+\hat{B} u$ with the sampling period $\Delta t=0.1$, where

$$
\hat{A}=\left[\begin{array}{cc}
0 & 1 \\
a_{1} & a_{2}
\end{array}\right], B=\left[\begin{array}{l}
0 \\
1
\end{array}\right], C=\left[\begin{array}{ll}
c_{1} & c_{2}
\end{array}\right] ;
$$

and $a_{1}, a_{2}, c_{1}, c_{2}$ are sampled from $\mathcal{N}(0,3)$ respectively. We check the relative degree to make sure that the system has relative degree 1 . The time horizon is taken as $N=40$. The "real " $\bar{Q}$ is generated by letting $\bar{Q}=\hat{Q} \hat{Q}^{T}$ where each elements of $\hat{Q}$ are sampled from the uniform distribution on $[-1,1]$. We set the feasible compact set for $Q$ as $\overline{\mathbb{S}}_{+}^{n}(5)$ and those randomly generated $\bar{Q}$ that does not belong to $\overline{\mathbb{S}}_{+}^{n}(5)$ is discarded. Each element of the initial conditions $\bar{x}^{(1: M)}$ are generated by sampling from a uniform distribution supported on $[-5,5]$. We generate 200 different sets of $(\hat{A}, \hat{B}, \bar{Q})$ and for each fixed $(\hat{A}, \hat{B}, \bar{Q}), 200$ trajectories are generated, i.e., $M=200$. 20dB of white Gaussian noises are added to $C x_{2: N}^{(1: M)}$ to get $y_{2: N}^{(1: M)}$. MATLAB function fmincon is used to solve the risk-minimizing problem. When solving the optimization problem, we use $Q=I$ as the initial iteration values for all cases. In the following we denote the estimation of $\bar{Q}$ as $Q_{\text {est }}$.

As illustrated in Fig. 1, the relative error $\| Q_{e s t}-$ $\bar{Q}\left\|_{F} /\right\| Q \|_{F}$ roughly decreases as $M$ increases. This empirically justifies Theorem III.1. 


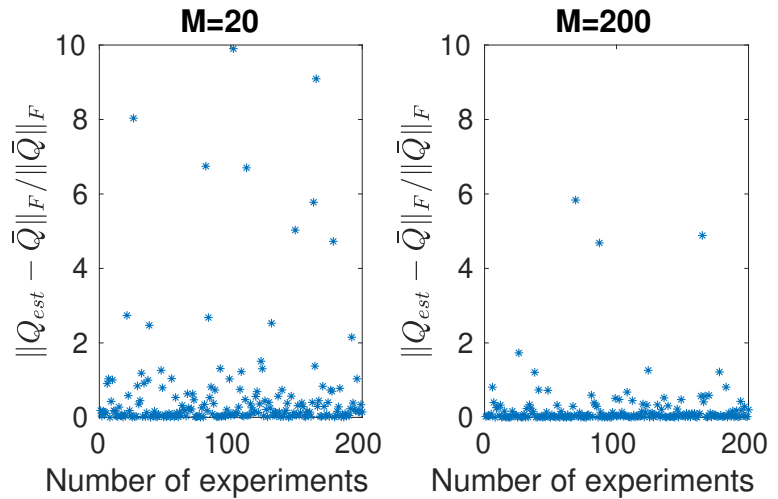

Fig. 1. The relative errors of the estimation by minimizing $\mathscr{R}_{M}(Q)$.

Next, we illustrate the performance of the estimation by using EM-algorithm under Gaussian assumptions. The groups of discrete-time linear systems $(A, B, C)$ and the "real" $\bar{Q}$ are generated in the same way as mentioned above. 100 groups for discrete-time linear systems are generated. For each system $(A, B, C)$, we generate 30 trajectories, i.e., $M=30$. The initial values are sampled from $\mathcal{N}\left(m_{1}=0, \Sigma_{1}=10 I\right)$. White Gaussian noises are added to $C x_{1: N}^{(1: M)}$ to get $y_{1: N}^{(1: M)}$ and $v_{t} \sim \mathcal{N}\left(0, \Sigma_{v}=0.01 I\right)$. We use $m_{1}=I, \Sigma_{1}=20 I$ and $Q=I$ as the initial values for the iterations in EM-algorithm for all groups of linear systems. The iterations are stopped when $\left\|Q_{k+1}-Q_{k}\right\|_{F} \leq 10^{-4}$. The results are shown as Fig. 2.

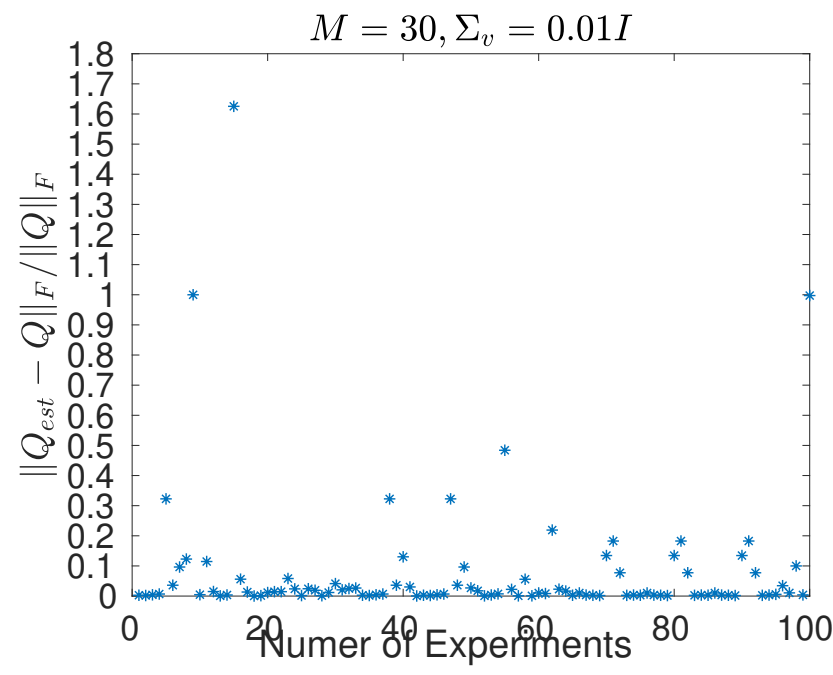

Fig. 2. The relative errors of the estimation by EM-algorithm under Gaussian assumption.

As it can be seen in Fig. 22 the relative error $\| Q_{\text {est }}-$ $\bar{Q}\left\|_{F} /\right\| Q \|_{F}$ is roughly small, while only a few of them is "huge". This is because EM-algorithm does not guarantee to find the global maximizer for the log-likelihood function and $Q_{k}$ converges to some local maxima.

\section{CONClusion}

In this paper, the problem of inverse optimal control for finite-horizon discrete-time LQRs is considered under the following two scenarios: 1) the distributions of the initial state and the observation noise are unknown, yet the exact observations on the initial states and the noisy observations on system output are available; 2 ) the exact observations on the initial states are not available, yet the observation noises are known white Gaussian and the distribution of the initial state is also Gaussian (with unknown mean and covariance). For the first scenario, the problem is formulated as a risk minimization problem and the statistical consistency for the estimation is proven. For the second scenario, we fit the problem into the framework of maximum-likelihood and EM-algorithm is used to solve this problem. We show the performance for the estimations empirically by numerical examples.

\section{REFERENCES}

[1] Brian DO Anderson and John B Moore. Optimal control: linear quadratic methods. Courier Corporation, 2007.

[2] Anil Aswani, Zuo-Jun Max Shen, and Auyon Siddiq. Inverse optimization with noisy data. arXiv preprint arXiv:1507.03266, 2015.

[3] Bastien Berret and Frédéric Jean. Why don't we move slower? the value of time in the neural control of action. Journal of neuroscience, 36(4):1056-1070, 2016.

[4] Dimitris Bertsimas, Vishal Gupta, and Ioannis Ch Paschalidis. Datadriven estimation in equilibrium using inverse optimization. Mathematical Programming, 153(2):595-633, 2015.

[5] Stephen Boyd, Laurent El Ghaoui, Eric Feron, and Venkataramanan Balakrishnan. Linear matrix inequalities in system and control theory, volume 15. Siam, 1994.

[6] Chelsea Finn, Sergey Levine, and Pieter Abbeel. Guided cost learning: Deep inverse optimal control via policy optimization. In International Conference on Machine Learning, pages 49-58, 2016.

[7] Takao Fujii. A new approach to the lq design from the viewpoint of the inverse regulator problem. IEEE Transactions on Automatic Control, 32(11):995-1004, 1987.

[8] Antony Jameson and Eliezer Kreindler. Inverse problem of linear optimal control. SIAM Journal on Control, 11(1):1-19, 1973.

[9] Robert I Jennrich. Asymptotic properties of non-linear least squares estimators. The Annals of Mathematical Statistics, 40(2):633-643, 1969.

[10] Wanxin Jin, Dana Kulić, Shaoshuai Mou, and Sandra Hirche. Inverse optimal control with incomplete observations. arXiv preprint arXiv:1803.07696, 2018

[11] Rudolf Emil Kalman. When is a linear control system optimal? Journal of Basic Engineering, 86(1):51-60, 1964.

[12] Arezou Keshavarz, Yang Wang, and Stephen Boyd. Imputing a convex objective function. In Intelligent Control (ISIC), 2011 IEEE International Symposium on, pages 613-619. IEEE, 2011.

[13] Timothy L Molloy, Jason J Ford, and Tristan Perez. Finite-horizon inverse optimal control for discrete-time nonlinear systems. Automatica, 87:442-446, 2018.

[14] Timothy L Molloy, Dorian Tsai, Jason J Ford, and Tristan Perez. Discrete-time inverse optimal control with partial-state information: A soft-optimality approach with constrained state estimation. In Decision and Control (CDC), 2016 IEEE 55th Conference on, pages 1926-1932. IEEE, 2016.

[15] Katja Mombaur, Anh Truong, and Jean-Paul Laumond. From human to humanoid locomotionan inverse optimal control approach. Autonomous robots, 28(3):369-383, 2010.

[16] Yurii Nesterov. Smoothing technique and its applications in semidefinite optimization. Mathematical Programming, 110(2):245-259, 2007.

[17] M Cody Priess, Richard Conway, Jongeun Choi, John M Popovich, and Clark Radcliffe. Solutions to the inverse lqr problem with application to biological systems analysis. IEEE Transactions on Control Systems Technology, 23(2):770-777, 2015.

[18] Torsten Söderström. Discrete-time stochastic systems: estimation and control. Springer Science \& Business Media, 2012.

[19] Mingxuan Sun and Danwei Wang. Analysis of nonlinear discrete-time systems with higher-order iterative learning control. Dynamics and Control, 11(1):81-96, 2001. 
[20] Aad W Van der Vaart. Asymptotic statistics, volume 3. Cambridge university press, 2000.

[21] Han Zhang, Jack Umenberger, and Xiaoming Hu. Inverse quadratic optimal control for discrete-time linear systems. arXiv preprint arXiv:1810.12590, 2018. 